\title{
LA-UR- 02-5237
}

Approved for public release;

dlstribution is unilimited.

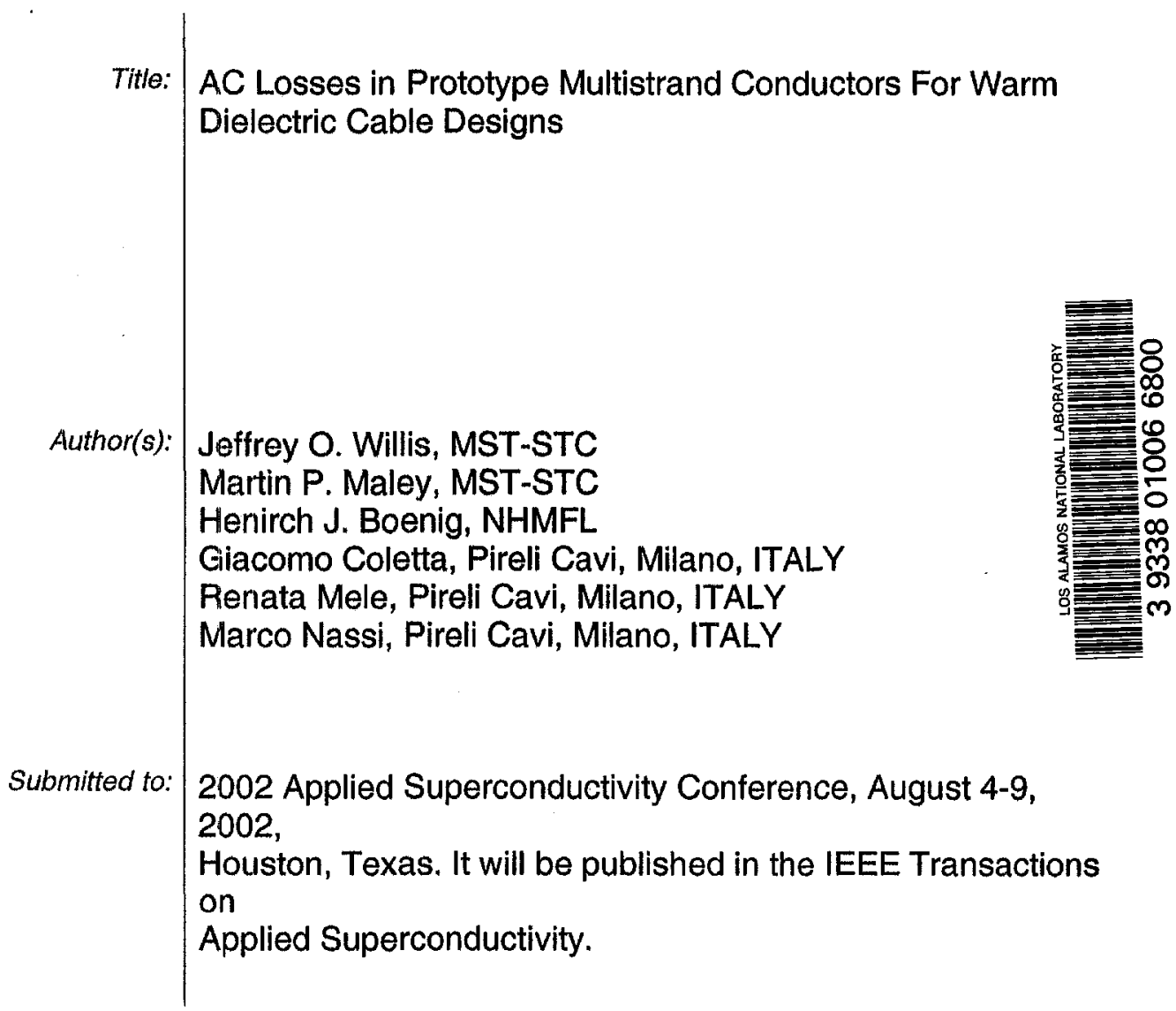

\section{7 \\ - Los Alamos \\ NATIONAL LABORATORY}

Los Alamos National Laboratory, an affirmative action/equal opportunity employer, is operated by the University of California for the U.S. Department of Energy under contract W-7405-ENG-36. By acceptance of this article, the publisher recognizes that the U.S. Government retains a nonexclusive, royalty-free license to publish or reproduce the published form of this contribution, or to allow others to do so, for U.S. Government purposes. Los Alamos National Laboratory requests that the publisher identify this article as work performed under the auspices of the U.S. Department of Energy. Los Alamos National Laboratory strongly supports academic freedom and a researcher's right to publish; as an institution, however, the Laboratory does not endorse the viewpoint of a publication or guarantee its technical correctness. 


\title{
Ac losses in Prototype Multistrand Conductors for Warm Dielectric Cable Designs
}

\author{
Jeffrey O. Willis, Member, IEEE, Martin P. Maley, Heinrich J. Boenig, Member, IEEE, Giacomo \\ Coletta, Renata Mele, and Marco Nassi
}

\begin{abstract}
We report on multiphase ac losses in four-layer prototype multi-strand conductors (PMCs) wound from HTS tape provided by American Superconductor Corporation. These conductors are prototypes warm dielectric cable designs, such as for the US Dept. of Energy's Superconductivity Partnership Initiative Project at Detroit Edison. We report on single phase "two phase"(no current in the PMC but with an external ac magnetic fleld generated by the two normal conductors arranged at the remaining corners of an equilateral triangle forming a three-phase configuration), and balanced three phase losses. Losses were also measured using a set of saddle coils to apply an ac magnetic field to the PMC. The losses were measured as a function of temperature, frequency, and current. We compare the losses for three PMCs, one (4LA) wound conventionally with equal pitch angles for all layers and the two others (4LB and $4 \mathrm{LC}$ ) wound to achieve equal current distribution (UCD) among the layers, and thus lower singlephase losses in the operating region. In addition, $4 \mathrm{LC}$ was wound with a newer generation tape having a higher critical current. The PMC 4LC was found to have the lowest single, twophase, and three-phase losses.
\end{abstract}

Index Terms- Superconducting power transmission lines, alternating current losses, multiphase losses, high temperature superconductors.

\section{INTRODUCTION}

S UPERCONDUCTING power transmission lines are being considered by the electric utility industry as a replacement and upgrade for existing oil-cooled copper undergroundtransmission lines in urban areas. In these areas, increasing loads require more current carrying capability. Because superconducting transmission lines can carry two to three times as much current as existing copper lines and can be

Manuscript received August 6, 2002. This work was supported in part by the U.S. Department of Energy, Office of Power Technologies, as part of the Superconductivity Program for Electric Systems.

J. O. Willis is with Los Alamos National Laboratory, MS-K763, Los Alamos, NM 87545 USA (telephone: 505-665-3030, fax: 505-665-3164, cmail: jwillis@lanl.gov).

M. P. Maley is with Los Alamos National Laboratory, MS-K763, Los Alamos, NM 87545 USA (c-mail: marty.maley@lanl.gov).

H. J. Boenig is retired from Los Alamos National Laboratory, MS-E536, Los Alamos, NM 87545 USA. His present address is 325 W. Burbank Fredericksburg, TX 78624 USA.

G. Coletta is with Pirelli Cavi e Sistemi, Viale Sarca 222, 20126 Milano Italy (telephone: +39-02-6442-9409, e-mail: giacomo.coletta@pirelli.com)

R. Mele is with Pirelli Cavi e Sistemi, Viale Sarca 222, 20126 Milano Italy (telephone: +39-02-6442-9410, e-mail: renata.mele@pirelli.com),

M. Nassi is with Pirelli Cavi e Sistemi, Viale Sarca 222, 20126 Milano Italy (e-mail: marco.nassi@pirelli.com). retrofitted into the same conduits, they offer a significant economic advantage in that they require no expensive new construction.

One of the most important issues in superconducting transmission line engineering design and application is the ac losses these conductors incur at power-line frequencies, both from the self magnetic field of the conductor, and, in certain designs, from the ac magnetic fields generated by the other two phases of a balanced three-phase line.

We report here on both the single phase and on multiphase ac losses resulting from the magnetic field of the other two phases of a three-phase superconducting power transmission line on the phase under test. There are several designs for retrofitting underground power transmission lines with high temperature superconductor (HTS) cables. In the "cold dielectric" design each superconducting phase is surrounded by a superconducting shield. Outside of the shield, therefore, there is no net magnetic field, so that there is no influence of one phase on the others. In the "warm dielectric" design there is no coaxial shield around each phase, thus reducing the use of HTS material significantly, but at the expense of interaction among the phases. The project led by NKT in Denmark employs a cable of the warm dielectric design [1]. If the conductors are relatively far apart, e.g., 20 to $30 \mathrm{~cm}$ or more, there is little effect of the fields from the other phase conductors [2]. This was the design of the Detroit Edison Superconductivity Partnership Initiative (SPI) cable retrofit project sponsored by the U.S. Department of Energy. Here, the major loss component is the single-phase loss with transport current flowing only in the PMC. However, for installations where space is at a premium, typically all three phase conductors are installed in a single conduit with conductor spacing on the order of $10 \mathrm{~cm}$, and the influence of the other phases does need to be considered.

We have measured the ac losses of three prototype multistrand conductors (PMCs) constructed as designs for retrofit cable projects. The cable is designed to carry 2400 Arms. The PMCs tested differ in tape critical current and in winding design. We will compare and contrast the performance of the conductor designs.

\section{EXPERIMENTAL}

\section{A. Experimental Apparatus and Procedure}

Voltage measurements have been used by a number of 
groups to measure the single-phase ac losses of power transmission line prototypes. However, complications arise as to placement of voltage contacts and interpretation of data when a PMC is subjected to external magnetic fields that may drive currents in circular paths that do not cause a voltage to appear at the contacts but do result in losses. This is in particular the case when the multiphase losses are to be measured in a PMC of the warm dielectric design.

For this reason, we chose to use a calorimetric technique for measuring the ac losses. Reference [3] contains substantial detail about the calorimeter construction and operation. Briefly, in this technique, the PMC is thermally isolated except at the two ends. Heat generated uniformly in the conductor results in a parabolic temperature profile along the conductor length. This profile is measured with platinum resistance thermometers and fitted to the equation:

$$
q_{L}=8 k A \Delta T_{m} / L^{2}
$$

where $q_{L}$ is the heat generation (loss) per unit length of the conductor, $k$ is the effective thermal conductivity along the length of the PMC, $A$ is the conductor cross-sectional area, $\Delta T_{m}$ is the temperature difference between the ends and the midpoint of the conductor, and $L$ is the conductor length. The factor $k A$ is determined independently by measuring the temperature gradient along the conductor when a known amount of power is generated by a bifilarly wound heater located at the midpoint. Because the determination of the ac-power loss is independent of the temperatures of the conductor ends, Joule heating at the normal/superconductor junction, which raises the temperature of the ends of the PMC, has little effect on the accuracy of the measurement. The loss is corrected for the temperature variation along the PMC [2].

The PMC is contained in a G-10 (glass epoxy) vacuum jacket with the ends (the current leads) cooled by the surrounding liquid nitrogen. The other two phases in the three-phase configuration are normal copper conductors, with the three phases arranged at the vertices of an equilateral triangle 10 or $20 \mathrm{~cm}$ on a side. Because the vacuum jacket of the PMC is a nonconductor, the PMC is affected by the magnetic field generated by the other two phases, similar to the environment present in a warm dielectric three-phase cable. The temperature of the liquid nitrogen bath can be varied from about $75 \mathrm{~K}$ to $64 \mathrm{~K}$ by controlling the pressure of the bath using a vacuum pump and pressure regulator.

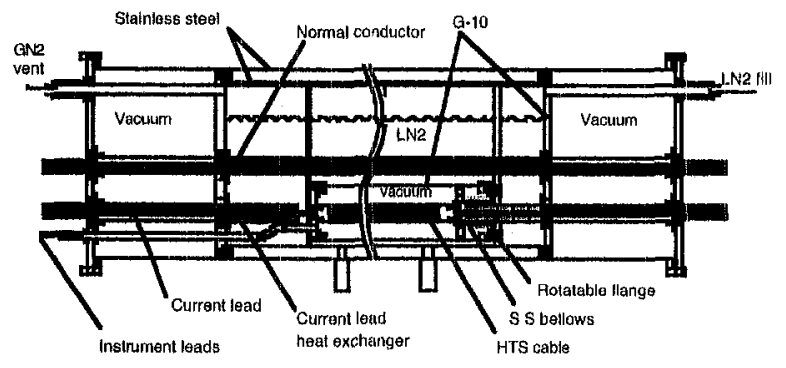

Fig.1. Schematic cross section of cable calorimeter.
TABLE I.

PMC PARAMETERS

\begin{tabular}{ccccc}
\hline PMC & $\begin{array}{c}\text { Tape } \mathrm{I}_{\mathrm{c}} \\
(\mathrm{A})\end{array}$ & $\begin{array}{c}\text { Cable } \mathrm{I}_{\mathrm{c}} \\
(\mathrm{A})\end{array}$ & $\begin{array}{c}\text { Gap } \\
(\mathrm{mm})\end{array}$ & Winding Angle \\
\hline 4LA & $60-85$ & 6000 & 0.1 & $\pm 30^{\circ}$ \\
4LB & $60-85$ & 5200 & 0.1 & variable \\
4LC & $90-100$ & 4800 & 1.5 & variable \\
\hline
\end{tabular}

Ac current is supplied by a standard, industrial-type $20 \mathrm{~kW}$ ac induction-motor drive modified for use as a variablefrequency power source. The output of the variable frequency source is connected to a $480 \mathrm{~V} / 5 \mathrm{~V}, 41.7 \mathrm{~A} / 4000 \mathrm{~A}$, threephase, step-down transformer connected in a delta/wye configuration. The power supply is typically operated over the frequency range of about $10 \mathrm{~Hz}$ to $180 \mathrm{~Hz}$. It can supply currents up to $3000 \mathrm{Arms}$ near $60 \mathrm{~Hz}$, but is limited to lower levels ( 1000 Arms) at low frequency by transformer core saturation and at high frequency by the transformer impedance. The current source performs well both under single-phase and balanced three-phase operation.

In the single-phase loss measurement configuration, ac current flows in the PMC, and the return path is a large distance $(>0.3 \mathrm{~m})$ from the PMC. In the "two-phase" configuration, current flows in the two normal phase conductors, generating a magnetic field at the location of the PMC, which carries no current. In the "three phase" configuration, balance, three-phase current flows in the two normal conductors and the PMC. Thus the PMC both carries a transport current and is subjected to a (rotating) magnetic field from the two other phase conductors.

\section{B. Cable}

The four layer prototype multistrand conductors (PMCs) were each wound by Pirelli from $\mathrm{Bi}-2223 / \mathrm{Ag}$ sheath multifilamentary tape manufactured by American Superconductor Corporation. PMC 4LA was wound conventionally with equal and opposite pitch angles in alternate layers of about 20 tapes each. PMCs 4LB and 4LC were wound to achieve the same inductance for each layer by adjustment of the pitch angle for each layer. This method was first proposed by the group at Siemens [4]. The result of this winding technique is to achieve uniform current distribution (UCD) among the layers of the PMC. PMC 4LC was wound using tape-to-tape spacing comparable to that achievable with commercial winding equipment and using a later generation, higher performance tape than was used in winding $4 \mathrm{LA}$ and 4LB. Each PMC is $1 \mathrm{~m}$ long and is wound on a $30 \mathrm{~mm}$ diameter core. Other parameters of the PMCs are listed in Table I. The critical current $I_{c}$ values were measured at $77 \mathrm{~K}$.

\section{RESULTS AND DISCUSSION}

A comparison of the single-phase losses of the PMCs at 76 $\mathrm{K}$ and $60 \mathrm{~Hz}$ is shown in Fig. 2. The losses depend on the current as a power law with an exponent $n$ near 3 . According to the uniform current distribution (UCD) model, magnetic flux penetrates into each of the four layers of PMCs $4 \mathrm{LB}$ and $4 \mathrm{LC}$ even at the lowest currents. In contrast, the monoblock model predicts that at low currents, flux will only penetrate the outer layers of PMC 4LA. Thus, the losses are lower for 
than $30^{\circ}$, the unbalanced area is larger than for $4 \mathrm{LA}$. However, these two PMCs show both larger (4LB) and smaller (4LC) two-phase losses than for $4 \mathrm{LA}$. This mechanism would also generate current flow in the end connectors, but there was no significant evidence of excess heating and temperature rise in those components. Thus, although the lack of systematic behavior among the PMCs suggests that this mechanism is not a major component of the two-phase losses, the presence of such an extrinsic effect cannot be ruled out.

Saddle-shaped magnetic field coils, which can apply a transverse magnetic field to the PMC, were added to the calorimeter system to extend the range and controllability of an applied field on the losses in the PMC. This effectively allows simulating a continuously varying spacing of the phase conductors. It was found in every case that the two-phase losses correlate directly with the strength of the applied ac magnetic field.

The balanced three phase losses of 4LC were also measured as a function of temperature, frequency, and spacing of the phase conductors. Fig. 5 shows a comparison of the single, two-phase, and balanced three-phase losses under one condition. It is clear that the single and two-phase loss magnitudes are not simply additive; the three-phase loss has a nonlinear dependence on the transport current and the applied magnetic field. All of the PMCs measured in the ac loss calorimeter should a similar nonlinear dependence, and PMC 4LC shows the best performance, i.e., the smallest ratio (three-phase loss/single-phase loss), about 2.7, of any of the four-layer PMCs. Three-phase losses twice as large were observed in many other PMCs correlating roughly with

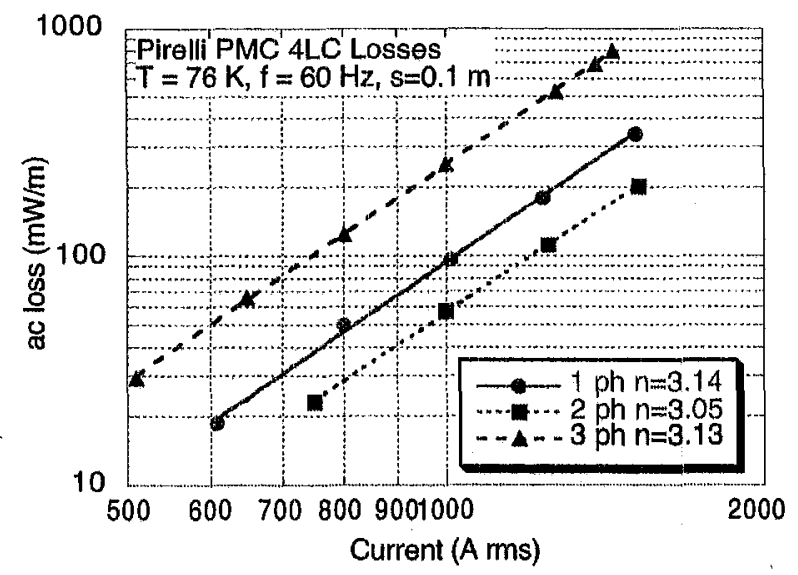

Fig. 5. Single, two-phase, and balanced three-phase losses of PMC 4LC at $76 \mathrm{~K}, 60 \mathrm{~Hz}$, and $0.1 \mathrm{~m}$ phase conductor spacing. The power law exponents $n$ are given in the legend. significantly larger two-phase losses. Thus control of the twophase loss appears to be important to achieve low three-phase losses.

\section{CONCLUSIONS}

We have measured the single, two-phase, and balanced three-phase losses of three prototype multistrand conductors for use in warm dielectric cables. The best performance was demonstrated for PMC 4LC, wound with adjusted pitch angles and of higher performance tape and with an inter tape spacing appropriate for machine winding. Single-phase losses at $50 \mathrm{~Hz}, 77 \mathrm{~K}$, and $2000 \mathrm{~A}$ were measured to be $0.69 \mathrm{~W} / \mathrm{m}$. The two phase losses were about $0.6 \mathrm{X}$ of this loss, and the three-phase losses, about $2.7 \mathrm{X}$. This is the best performance of any PMC measured in the ac loss calorimeter, and should be suitable for a warm dielectric cable conductor application.

\section{ACKNOWLEDGMENT}

The authors wish to thank Prof. John Clem and Drs. Masaki Suenaga and Stephen Ashworth for stimulating discussions throughout the course of this work.

\section{REFERENCES}

[1] D. Willen et al., "First operation experiences from a $30 \mathrm{kV}, 104 \mathrm{MVA}$ HTS power cable installed in a utility substation," Physica $C$ (in press)

[2] D. E. Daney, H. J. Boenig, M. P. Maley, J. Y. Coulter, S. Fleshler, "Single and three-phase ac losses in HTS superconducting power transmission line prototype cables," in Advances in Cryogenic Engineering (Materials), vol. 44, U. Balachandran, et al., Eds. New York: Plenum Press, 1998, p. 791.

[3] D. E. Daney, H. J. Boenig, M. P. Maley and S. Fleshler, "Calorimeter for measuring AC losses in HTS cables for power transmission lines," Cryogenics vol. 39, pp. 225- 234, Mar. 1999.

[4] G. Ries, M. Leghissa, J. Rieger, J. Wiezorek, and M. Oomen, "High-T superconductors and ac loss in electrotechnical devices," Physica C, vol. 310, pp. 283-290, Dec. 1998; J. Rieger, M. Leghissa, J. Wiezoreck, H.-P. Krämer, G. Ries, and H.-W. Neumüller, "Development of a $10 \mathrm{~m}$ long superconducting multistrand conductor for power transmission cables, Supercond. Sci. Technol., vol. 11, pp. 902-8, Sept. 1998.

[5] W. T. Norris, "Calculation of hysteresis losses in hard superconductors cartying ac: isolated conductors and edges of thin sheets," $J$. Phys. $D$ vol. 3, pp, 489-507, Apr. 1970.

[6] J. O. Willis, D. E. Daney, M. P. Maley, H. J. Boenig, R. Mele, G. Coletta, and M. Nassi, "Coupling losses in HTS prototype multistrand conductors as a function of frequency and magnitude of external ac magnetic field," in Advances in Cryogenic Engineering (Materials), vol. 46, U. Balachandran, D. U. Gubser, K. T. Hartwig, and V. A. Bardos, Eds. New York: Kluwer Academic/Plenum Publishers, 2000, p. 623-629.

[7] J. O. Willis, D. E. Daney, M. P. Maley, H. J. Boenig, R. Mele, G. Coletta, M. Nassi, and J, R. Clem, "Multiphase ac loss mechanisms in prototype multistrand conductors," IEEE Trans. Appl. Supercond. vol. 11 , pp. 2188-92, March 2001.

[8] M. N. Wilson, "Superconducting Magnets," New York: Oxford University Press, 1983.

[9] J, R, Clem, private communication, June 2000. 